\title{
IMPROVING END-OF-TURN DETECTION IN SPOKEN DIALOGUES BY DETECTING SPEAKER INTENTIONS AS A SECONDARY TASK
}

\author{
Zakaria Aldeneh $^{1}$, Dimitrios Dimitriadis ${ }^{2 *}$, Emily Mower Provost ${ }^{1}$ \\ ${ }^{1}$ University of Michigan at Ann Arbor, ${ }^{2}$ Microsoft \\ \{aldeneh, emilykmp\}@umich.edu, didimit@microsoft.com
}

\begin{abstract}
This work focuses on the use of acoustic cues for modeling turn-taking in dyadic spoken dialogues. Previous work has shown that speaker intentions (e.g., asking a question, uttering a backchannel, etc.) can influence turn-taking behavior and are good predictors of turn-transitions in spoken dialogues. However, speaker intentions are not readily available for use by automated systems at run-time; making it difficult to use this information to anticipate a turn-transition. To this end, we propose a multi-task neural approach for predicting turntransitions and speaker intentions simultaneously. Our results show that adding the auxiliary task of speaker intention prediction improves the performance of turn-transition prediction in spoken dialogues, without relying on additional input features during run-time.
\end{abstract}

Index Terms - Multi-task learning, recurrent neural networks, LSTM, turn-taking, spoken dialogues, speaker intentions

\section{INTRODUCTION}

Dialogue agents must be able to engage in human-like conversations in order to make interactions with spoken dialogue systems more natural and less rigid. Turn-management is an essential component of conversations as it allows participants in a dialogue to exchange control of the floor. Studies have shown that conversation partners rely on both syntactic and prosodic cues to anticipate turn-transitions [1, 2, 3]. Syntactic cues include keywords and semantics of an uttered sentence. Prosodic cues include the final intonation of a clause, pitch level, and speaking rate. In this work, we assess the efficacy of using acoustic cues for anticipating turn-switches in dyadic spoken dialogues. Given a single utterance, our goal is to use acoustic cues to predict if there will be a switch in speakers for the upcoming utterance or not.

Modern spoken dialogue systems generally rely on simple thresholding approaches for modeling turn-taking [4, 5, 6]. However, turn-management is a complex phenomenon, in which participants in a conversation rely on multiple cues

${ }^{*}$ Work completed while at IBM T. J. Watson Research Center. to anticipate turn changes or end-of-turns. We anticipate that interactions between humans and machines can be improved if dialogue systems can accurately anticipate turn-switches in spoken conversations.

Turn-taking in conversations can take many forms. The two basic turn-taking functions are hold and switch. Given an utterance in a conversation, a hold indicates that the next utterance will be uttered by the same speaker while a switch indicates that the next utterance will be uttered by the other speaker in the conversation. Turn-switches can be further divided into smooth and overlapping switches [3]. Smooth switches occur when there is silence between two consecutive utterances from two speakers. Overlapping switches occur when a speaker starts uttering a sentence before the other speaker finishes uttering his/her sentence.

Previous works built models that used both acoustic and syntactic information to anticipate turn-changes to help make turn-management more natural in spoken dialogue systems [3], 5]. Gravano and Hirschberg showed that raising contours of intonation correlates with turn-transitions while flat intonations correlates with turn-holds [3]. They also showed that certain keywords (e.g., "you know...") and textual completion have good correlations with turn-management functions. In addition to the usefulness of syntactic and acoustic cues for modeling turn-taking, previous work showed that speaker intentions (e.g., ask a question, utter a backchannel, etc.) can be good predictors of turn-transitions in dialogues [7, 8]. For example, a switch in speaker turns is more likely to occur after encountering a question than it is to occur after encountering a statement. Although speaker intentions (sometimes referred to as dialogue acts) are useful for predicting turntransitions [7, 8], they require human annotations and are not readily available during run-time.

We propose the use of a multi-task Long Short-Term Memory (LSTM) network that takes in a sequence of acoustic frames from a given utterance and predicts turn-transitions and speaker intentions simultaneously. During training time, the network is optimized with a joint loss function using ground-truth labels for turns and intentions. During test time, the network makes two predictions, one of which can be discarded or used by other modules in a spoken dialogue system. The advantage is that this allows the model to use represen- 
tations that encode information about speaker intentions for anticipating turns changes. Our experiments demonstrate that adding the detection of speaker intentions as a secondary task improves the performance of anticipating turn-transitions.

\section{RELATED WORK}

The problem of modeling turn-taking in conversations has been extensively studied in the literature. In this section, we give an overview of related works that focused on speech or textual interactions (i.e., no visual cues). Our work complements previous work by showing that a model that uses acoustic cues for predicting turn-switches benefits from adding speaker intentions prediction as an auxiliary task using the multi-task learning framework.

One line of work looked at the use of acoustic and lexical features for modeling turn-taking behavior [4, 5, 9, 10]. Liu et al. [5], Masumura et al. [9], and Ishimoto et al. [10] looked at the problem in Japanese conversations while Maier et al. [4] looked at the problem in German conversations. Masumura et al. proposed using stacked time-asynchronous sequential networks for detecting end-of-turns given sequences of asynchronous features (e.g., MFCCs and words) [9]. Ishimoto et al. investigated the dependency between syntactic and prosodic features and showed that combining the two features is useful for predicting end-of-turns [10]. Liu et al. built a Recurrent Neural Network (RNN) to classify a given utterance into four classes that relate to turn-taking behavior using joint acoustic and lexical embeddings [5]. Finally, Maier et al. built an LSTM with a threshold-based decoding and studied the trade-off between latency and cut-in rate for end-of-turn detection in simulated real-time dialogues [4]. The conclusion reached by this line of work was that end-of-turn detection models benefit from augmenting classifiers that use acoustic information with lexical information.

Another line of work focused solely on the acoustic modality, pointing out that using lexical features would (1) require access to a speech recognition pipeline and (2) bias the classifiers due to varying prompt types [11]. Arsikere et al. compared the effectiveness of acoustic features (e.g., pitch trends, spectral constancy, etc.) for predicting end-ofturns in two datasets that differed in prompt type (one is slow and deliberate, the other is fast and spontaneous) [11]. They found that the same acoustic cues were useful for detecting end-of-turns for both prompt types.

A final line of work used dialogue act information when modeling turn-taking behavior [7, 8, 12]. Guntakandla and Nielsen built a turn-taking model that relied on transcribed segments, intention labels, speaker information, and change in speaker information to predict turn-transitions in dialogues [8]. Meshorer and Heeman used current and past speaker intention labels along with two new features, relative turn length and relative floor control, summarizing past speaker behavior for predicting turn-switches in dia- logues [7]. Finally, Heeman and Lunsford showed that turntaking behavior not only depends on previous and upcoming speech act types, but also depends on the nature of a dialogue; suggesting that turn-taking events should be split into several groups depending on speech act types and the context of the dialogue [12].

The works of Meshorer and Heeman [7], Guntakandla and Nielsen [8], and Heeman and Lunsford [12] suggested that speaker intentions can be useful for predicting turntransitions. However, speaker intentions are not readily obtainable from utterances and require manual human annotations. We are interested in studying how we can augment acoustic systems with speaker intention information, available during training time, to improve performance of turn-transitions predictions.

\section{PROBLEM SETUP}

We follow the work of Meshorer and Heeman [7] and represent a conversation between two speakers as a sequence of utterances, taking the following form:

$$
u_{1}, u_{2}, \ldots, u_{N}
$$

where each $u_{i}$ is an utterance in the conversation. The sequence of utterances are sorted in terms of start talk time. Let $\operatorname{spkr}(\cdot)$ be a function that returns the speaker of a given utterance. Given $u_{i}$, the goal is to predict whether the following statement is true or false:

$$
\operatorname{spkr}\left(u_{i}\right) \neq \operatorname{spkr}\left(u_{i+1}\right)
$$

If the statement is true, then a turn-switch will take place and the other speaker will speak next. If the statement is false, then the current speaker will continue speaking.

Each utterance in the sequence represents a complete sentence, containing both acoustic and lexical cues, and varies in duration. We assume that we know the end-points of each utterance as in [7, 8, 11]. Utterance end-points can be readily obtained from modern voice-activity detection algorithms or using an end-of-utterance detection systems (e.g., [6]). We leave the problem of combining our multi-task model with end-of-utterance detection for future work and focus on the problem of predicting turn-switches from acoustic cues for a given utterance.

\section{DATASET AND FEATURES}

\subsection{Dataset}

We use the Switchboard corpus [13] to model turn-taking behavior in spoken dialogues. The corpus consists of dyadic telephony conversations between participants who were asked to discuss various topics. We use the annotations provided 
Table 1: Mapping dialogue act classes to intention classes.

\begin{tabular}{ll}
\hline SwDA classes & Intention classes \\
\hline sd, h, bf & statement \\
sv, ad, sv@ & opinion \\
aa & agree \\
$\%, \%-$ & abandon \\
b, bh & backchannel \\
qy, qo, qh & question \\
no, ny, ng, arp & answer \\
\hline
\end{tabular}

by the Switchboard Dialog Act Corpus (SwDA), since we are interested in utilizing speaker intentions (i.e., dialogue act types). The goal of SwDA corpus was to extend the original Switchboard corpus with dialogue act types that summarize turn information in the conversations.

However, the SwDA corpus does not map dialogue acts to timing information in the original media files of the Switchboard corpus. It only maps dialogue acts to lexical and turn information. We augment the SwDA corpus with the NXT Switchboard corpus [14] to get utterance timing information from the original media files. The aim of the NXT Switchboard corpus was to combine major annotations that were performed on the Switchboard corpus and make them accessible within one framework.

Preparation. We first add binary turn labels (switch/hold) to each utterance in the dataset. We focus on 7 major dialogue acts which we obtain by grouping different SwDA classes as shown in Table 1 The dialogue act groups that we use are a subset of those used in [7]. We filtered out utterances in the dataset that do not have corresponding audio segments (i.e., no timing information). We obtain the final utterances by trimming the audio of the appropriate speaker channel in the original media files in accordance to the timings provided in the NXT Switchboard corpus.

Analysis. The final dataset that we use contains a total of 86,687 utterances. Table 2 shows a summary of the content of the dataset in terms of turn labels and speaker intentions. As a first pass to understand the relationship between dialogue acts and turns, we run a Chi-square test of independence and find that there is a relationship between dialogue acts and turns, $p<0.001$ (i.e., they are not independent). Note that this finding is suggestive rather than conclusive; mainly because our utterances are not independent (they can come from same speaker). Nevertheless, this finding supports findings in literature [3, 12], which suggested that speaker intentions influence turn-taking behavior.

\subsection{Features}

We use the OpenSMILE toolkit [15] to extract the following features and their first (left) derivatives using a $25 \mathrm{~ms} \mathrm{Ham}-$

\footnotetext{
${ }^{1}$ https://github.com/cgpotts/swda
}

Table 2: Total number of utterances that are followed by holds and switches for each speaker intention class.

\begin{tabular}{ccc}
\hline \multirow{2}{*}{ Intention } & \multicolumn{2}{c}{ Counts $(\%)$} \\
& Holds & Switches \\
\hline statement & $26,332(52.2)$ & $12,722(35.1)$ \\
opinion & $8,066(16.0)$ & $5,227(14.4)$ \\
agree & $3,997(7.9)$ & $1,417(3.9)$ \\
abandon & $3,887(7.7)$ & $3,203(8.8)$ \\
backchannel & $6,225(12.3)$ & $10,678(29.5)$ \\
question & $752(1.5)$ & $2,369(6.5)$ \\
answer & $1,197(2.4)$ & $615(1.7)$ \\
total & 50,456 & 36,231 \\
\hline
\end{tabular}

ming window with a shift-rate of $10 \mathrm{~ms}$ : intensity, loudness, MFCC, RMS energy, zero-crossing-rate, and smoothed pitch. As a result, a given signal is represented as a sequence of 42-dimensional feature vectors. The choice of these features was inspired by their success in previous studies on modeling turn-taking using acoustic cues [4, 11].

\section{METHOD}

We use unidirectional LSTM network to model the sequence of acoustic features and make turn predictions. LSTMs are able to capture past signal behavior and they have shown success in many audio processing applications, such as speech recognition and computational paralinguistics [6, 16]. In addition to their ability to capture past signal behavior, LSTMs are able to capture information relating to timing and differentials (e.g., rising slope); both of which are useful for modeling turn-taking [3].

Predicting turns can be formulated as a binary classification task where the goal is, given an utterance, predict whether there will be a turn-switch or a turn-hold. We augment this task given the following problem setup: given an utterance, simultaneously predict turn-transitions and speaker intentions. The model is trained to minimize a joint loss function that takes the following form:

$$
L_{t o t}=\lambda_{1} L_{t u r n}+\lambda_{2} L_{\text {intent }}
$$

where $L_{t u r n}$ is the loss function for turn predictions, $L_{\text {intent }}$ is the loss function for speaker intention predictions, $L_{t o t}$ is the overall loss function, $\lambda_{1}$ and $\lambda_{2}$ are weights assigned to control the influence of each loss function. In this work we set $\lambda_{1}$ to 1.0 and $\lambda_{2}$ to 0.5 .

Baselines. We re-implement the "full model" from [7] and compare its performance to the proposed approach. The full model uses a Random Forest classifier with the features described in Section 2 . We also compare our proposed multitask approach to a single-task LSTM that is trained to minimize $L_{\text {turn }}$ alone. We note that the single-task LSTM approach is similar to the one used in [4]. 
Table 3: Performance comparison of different methods. Results shown are macro-averages across turn-switches and turn-holds.

\begin{tabular}{lcccc}
\hline Method & Rec. & Prec. & F1 & AUC \\
\hline Random & 50.0 & 49.6 & 41.7 & 45.3 \\
Full model [7] & 55.8 & 56.7 & 55.4 & 57.8 \\
LSTM & 65.9 & 65.6 & 65.5 & 71.9 \\
MT-LSTM & $\mathbf{6 6 . 4}^{*}$ & $\mathbf{6 6 . 0}$ & $\mathbf{6 5 . 8}$ & $\mathbf{7 2 . 6}^{*}$ \\
\hline * indicates $p<0.05$ under a paired $t$-test with LSTM.
\end{tabular}

\section{EXPERIMENTS}

\subsection{Setup}

We evaluate performance using 5-fold cross-validation. We split on conversations, as opposed to utterances, to ensure that individual speakers do not appear in both the training and testing folds. For each testing fold, we randomly take out $33 \%$ of the training conversations and use them for validation and early stopping. For each conversation, we perform speakerspecific $z$-normalization on the features.

We implement our models using the PyTorch library2. We optimize the weighted negative log-likelihood loss function and use RMSProp optimizer to train our models. We use an initial learning rate of 0.001 . At the end of each epoch, we compute the macro-F1 score on the validation set and reduce the learning rate by a factor of 2 if there was no improvement from last epoch. We run for a maximum of 100 epochs and stop training if there was no improvement in validation F1 score for 5 consecutive epochs. We take a snapshot of the model after each epoch and select the one that gave the highest validation performance.

For each fold, we perform a grid search and pick the hyper-parameters that maximize validation performance. The main hyper-parameters of the model are: number of layers $\{1,2\}$ and layer width $\{32,64,128\}$. Once we have identified the optimal hyper-parameters for each fold, we train 3 models with different random seeds and report their ensemble performance to minimize variance due to random initialization. We report the average performance across the five folds.

\subsection{Results and Discussion}

Table 3 shows the results obtained from our experiments. The table shows that a single-task LSTM, which uses the input features described in Section 4.2, outperforms the full model in all evaluation metrics. We attribute this improvements to better feature representations and better sequential modeling abilities of LSTMs. The table shows that a multi-task LSTM, which is trained using a joint loss function, provides consistent improvements over a single-task LSTM (significant improvements under a paired $t$-test, $p<0.05$, in terms of recall

\footnotetext{
${ }^{2}$ https://github.com/pytorch/pytorch
}

Table 4: Detecting turn-switches and turn-holds for each speaker intention class, as well as the per-class accuracy for detecting intentions by the auxiliary task.

\begin{tabular}{cccc}
\hline & \multicolumn{2}{c}{ Turn-Transitions } & Intentions \\
& F1 (switch) & F1 (hold) & per-class Acc. \\
\hline statement & 51.4 & 73.2 & 39.6 \\
opinion & 54.5 & 70.3 & 43.1 \\
agree & 49.7 & 66.5 & 30.4 \\
abandon & 67.2 & 68.3 & 56.8 \\
backchannel & 79.1 & 51.6 & 49.6 \\
question & 72.2 & 47.6 & 50.3 \\
answer & 61.1 & 71.7 & 43.9 \\
\hline
\end{tabular}

and AUC). This suggests that a turn prediction model can benefit from representations extracted for detecting speaker intentions. Next, we study how well our model is able to identify turn-switches when the switches are smooth and when they are overlapping. Our model identifies turn-switches with a recall of $68.5 \%$ when the switches are overlapping and identifies turn-switches with a recall of $68.1 \%$ when the switches are smooth.

Table 4 shows the performance of predicting turn-switches and turn-holds for each intention class, as well as the accuracy of detecting that intention class. The results show that the model is better able to predict turn-switches when presented with a backchannel or a question, and is better able to predict turn-holds when presented with a statement, opinion, or an answer. This suggests that the performance of the model depends on the context and nature of a dialogue, and that it is easier to anticipate turn-switches or turn-holds for some intentions and not for others.

Table 4 also shows the performance of identifying speaker intentions by the auxiliary task. The table shows that it is easier to identify backchannels, questions, or turn-exit signals (abandon) than it is to identify agreement signals and statements. The auxiliary task obtains an unweighted average recall (UAR) of $45.6 \%$ on a 7 -way classification task (where chance UAR is $14.3 \%$ ).

\section{CONCLUSION}

In this work we showed that a model that uses acoustic features for modeling turn-taking in spoken dialogues could benefit from adding speaker intention detection as an auxiliary task. We also explored how the performance of our turntaking model varies depending on speaker intentions. For future work, we plan to augment acoustic features with lexical or phonetic information. We also plan to investigate combining turn-taking with end-of-utterance detection. Finally, we plan to add our model to a live spoken dialogue system.

Acknowledgment. This work was supported by IBM under the Sapphire project. 


\section{REFERENCES}

[1] Stephen C Levinson, "Turn-taking in human communication-origins and implications for language processing," Trends in cognitive sciences, vol. 20, no. 1, pp. 6-14, 2016.

[2] Simon Garrod and Martin J Pickering, "The use of content and timing to predict turn transitions," Frontiers in psychology, vol. 6, pp. 751, 2015.

[3] Agustín Gravano and Julia Hirschberg, "Turn-taking cues in task-oriented dialogue," Computer Speech \& Language, vol. 25, no. 3, pp. 601-634, 2011.

[4] Angelika Maier, Julian Hough, and David Schlangen, "Towards deep end-of-turn prediction for situated spoken dialogue systems," Interspeech, 2017.

[5] Chaoran Liu, Carlos Ishi, and Hiroshi Ishiguro, "Turntaking estimation model based on joint embedding of lexical and prosodic contents," Interspeech, pp. 16861690, 2017.

[6] Carolina Parada, Gabor Simko, Matt Shannon, and Shuo-yiin Chang, "Improved end-of-query detection for streaming speech recognition," pp. 2900-2904, 2017.

[7] Tomer Meshorer and Peter A Heeman, "Using past speaker behavior to better predict turn transitions.," in Interspeech, 2016, pp. 2900-2904.

[8] Nishitha Guntakandla and Rodney Nielsen, "Modelling turn-taking in human conversations," in $A A A I$ Spring Symposium on Turn-Taking and Coordination in Human-Machine Interaction, Stanford CA, 2015.

[9] Ryo Masumura, Taichi Asami, Hirokazu Masataki, Ryo Ishii, and Ryuichiro Higashinaka, "Online end-ofturn detection from speech based on stacked timeasynchronous sequential networks," Interspeech, pp. 1661-1665, 2017.

[10] Yuichi Ishimoto, Takehiro Teraoka, and Mika Enomoto, "End-of-utterance prediction by prosodic features and phrase-dependency structure in spontaneous japanese speech," Interspeech, pp. 1681-1685, 2017.

[11] Harish Arsikere, Elizabeth Shriberg, and Umut Ozertem, "Enhanced end-of-turn detection for speech to a personal assistant," in AAAI Spring Symposium on Turn-taking and Coordination in Human-Machine Interaction, 2015.

[12] Peter A Heeman and Rebecca Lunsford, "Turn-taking offsets and dialogue context," Interspeech, pp. 16711675, 2017.
[13] John J Godfrey, Edward C Holliman, and Jane McDaniel, "Switchboard: Telephone speech corpus for research and development," in Acoustics, Speech, and Signal Processing, 1992. ICASSP-92., 1992 IEEE International Conference on. IEEE, 1992, vol. 1, pp. 517520 .

[14] Sasha Calhoun, Jean Carletta, Jason M Brenier, Neil Mayo, Dan Jurafsky, Mark Steedman, and David Beaver, "The nxt-format switchboard corpus: a rich resource for investigating the syntax, semantics, pragmatics and prosody of dialogue," Language resources and evaluation, vol. 44, no. 4, pp. 387-419, 2010.

[15] Florian Eyben, Martin Wöllmer, and Björn Schuller, "Opensmile: the munich versatile and fast open-source audio feature extractor," in Proceedings of the 18th ACM international conference on Multimedia. ACM, 2010, pp. 1459-1462.

[16] Raymond Brueckner, Maximilian Schmitt, Maja Pantic, and Björn Schuller, "Spotting social signals in conversational speech over ip: A deep learning perspective," Interspeech, pp. 2371-2375, 2017. 